\title{
On the Forecasting of the Movement of Typhoon
}

\author{
by
}

\author{
Y. Masuda, M. Takeuchi and M. Hashimoto
}

Meteorological Research Institute

(Received December 26, 1952)

\begin{abstract}
From the results of streamline analyses of typhoons, it is found that the point which corresponds to Scherhag's "delta" appears in the moving direction of typhoon, and approaches to the typhoon center with height. And the fact that the direction from the typhoon center to this delta point agrees with the direction of a steering current and typhoon moves along this direction, is clarified.
\end{abstract}

Theoretical studies for the typhoon movement are treated by many authors [1], [2], [3], and the fact that the well-known empirical law that "the typhoon moves with a steering current" holds approximately is shown. Hawever, in reality, precise selection of a steering current is difficult. T. OOTANI [4], vectorially adding the wind velocities at two points symmetrical with respect to the typhoon center, computed the steering current. However, as the opportunity to obtain the wind data of such two points at the same time is rare, and also, as the observational values of wind fluctuate in a large degree, this method has some difficulty to use practically. G. Norton [5] drew the streamlines near

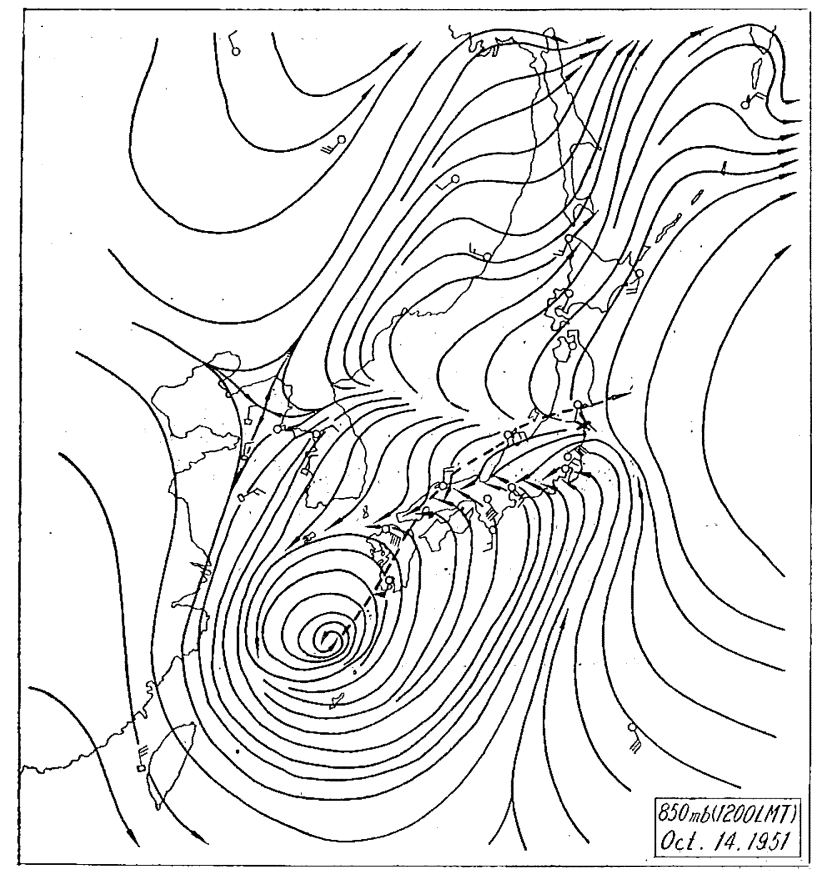

(a) 


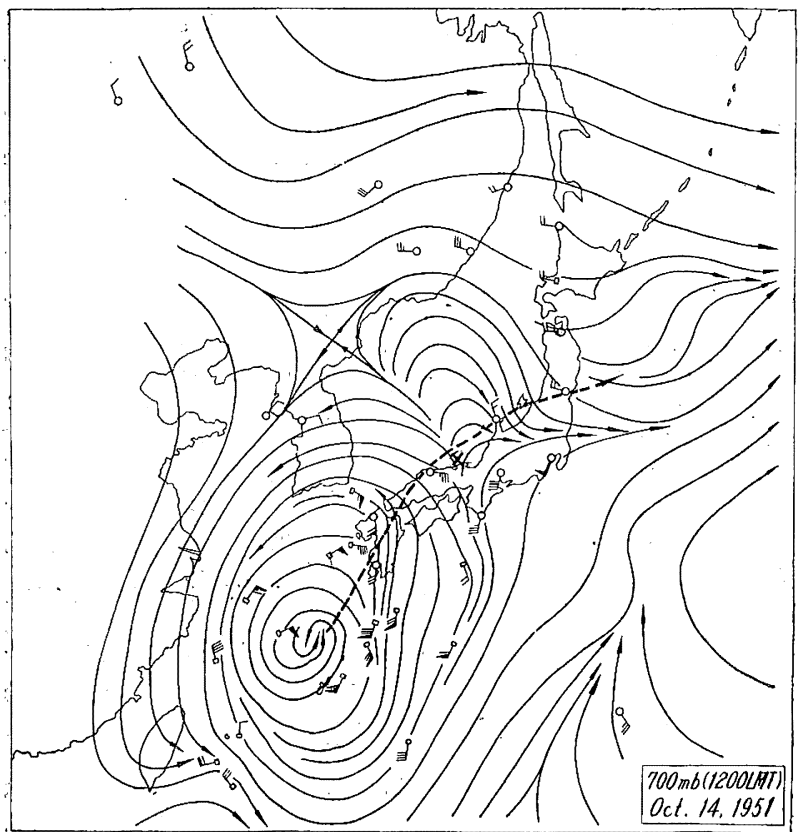

(b)

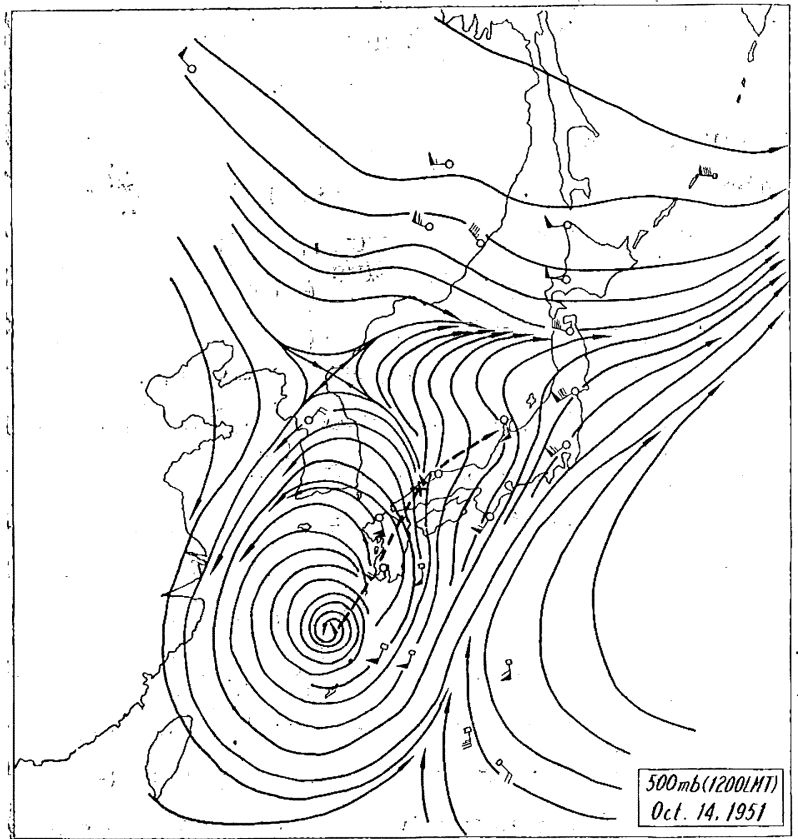

(c)

Fig. 1. Representative patterns of streamlines in the case of the typhoon Ruth. In these figures, the solid lines represent stream lines, the heavy dashed lines the typhoon track and the symbols $x$ the delta points respectively. Barbs show the representative ones of wind observation data. the typhoon, and showed that the level on which the typhoon circulation disappears is the steering level, and the typhoon moves with the wind just above the typhoon center on that level. And he fairly succeeded. But the levels on which the typhoon circulation disappears differ with the typhoon scale, and it is difficult to detect the steering level for each individual case. L. SHERMAN [6], using the two wind observations at the same radial distance from the hurricane center, introduced the method of computing the direction and the velocity of the steering current, and obtained a correlation of about 0.70 between computed orientations of the translatory wind components and the observed direction of the propagation of hurricane centers. However, this method also has the same fault as OotANI's. M. Hashimoto [7], applying Syono's theory [2], obtained pressure force acting in an appropriate circle around a typhoon, and from this force estimated the direction and velocity of a steering current. This method also has a weakness that by this method only an instantaneous direction and velocity of a steering current are estimated, and various re- 


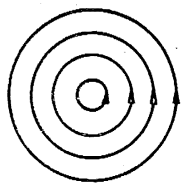

(a)

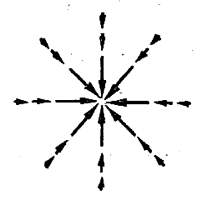

(b)

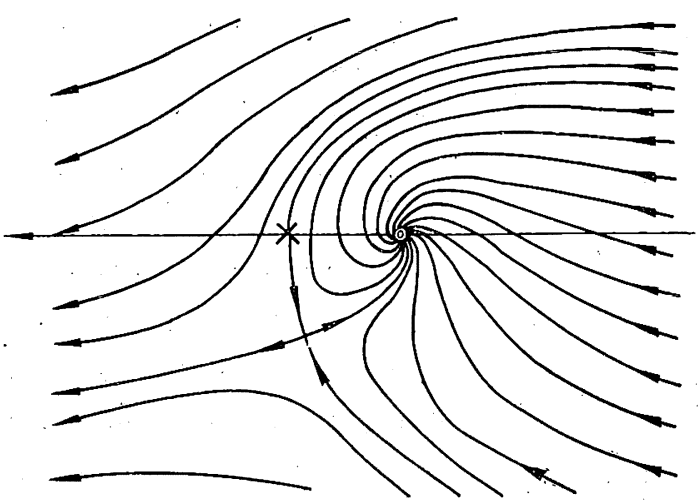

(d)

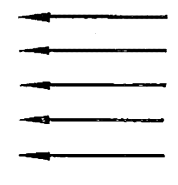

(c) sults are obtained according. to what circle around the typhoon is selected.

From the streamline analyses for the typhoon Ruth, an interesting result is obtained. Fig. 1. is one of the representative charts of streamlines in the case of the typhoon Ruth. In this figure, stream. lines are drawn by Sandström's method using wind data of rawin and pibal for each aerological station, and of all aerial weather reconnaissances: available. However, intervals: between two adjacent streamlines are not inversely proportional to wind velocities, but are taken arbitrarily. So, from this figure directly, it is.

unable to compute divergence and convergence.

From this figure, the characteristic features of streamlines in the vicinity of at typhoon are known. That is, in the neighbourhood of the typhoon center, all streamlines curve with a cyclonic curvature and direct towards the center spirally. This fact suggests that the winds in the neighbourhood of the typhoon center are composed of radial components towards the center and tangential components around the center cyclonically. If we turn our attention to the moving direction of the typhoon, we may find the characteristic point (symbol $x$ in this figure), inside which the streamlines: direct towards the center with a cyclonic curvature and outside which they go out with an anticyclonic one. That is to say, from this point, the streamlines spread out. just in the shape of Scherhag's "delta". So we name it as the delta point.

The fact that the delta point appears in the moving direction of typhoon, suggests: that the uniform steering current superposes upon the wind system of the typhoon. That is, as L. SHerman [6] showed, we may think that the wind system of the typhoon. is composed of radial components towards the center, and of the tangential components around the center cyclonically, and the uniform steering current. The streamlines of this wind system is shown in Fig. 2. At the delta point (symbolx), the absolute values of the steering current and the radial component towards the center are equal, and the directions of those compontents are inverse to each gther. In the 


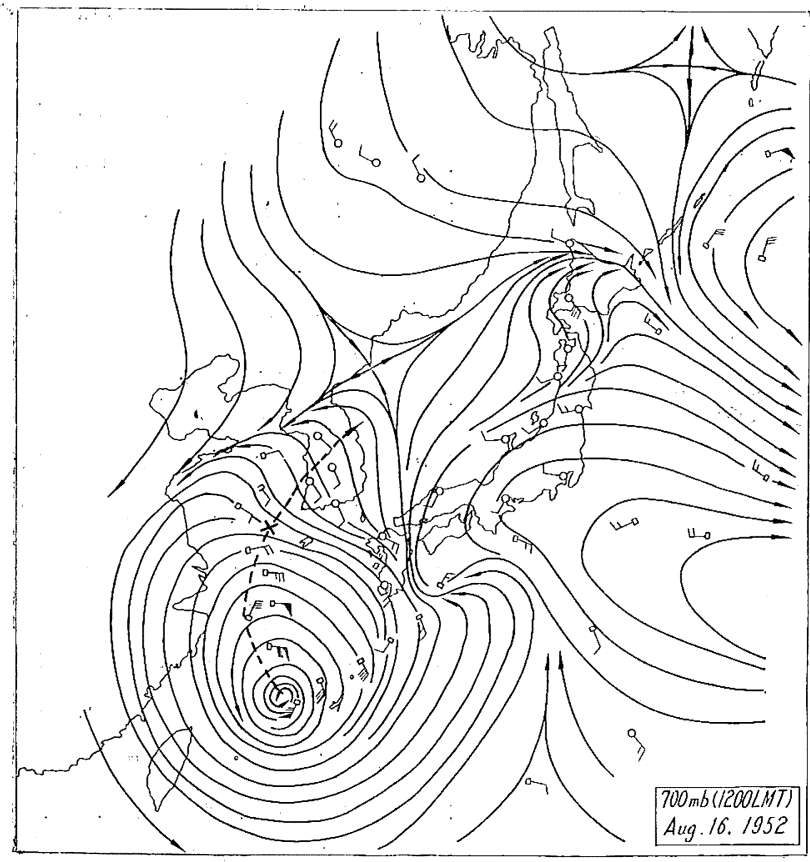

(a)

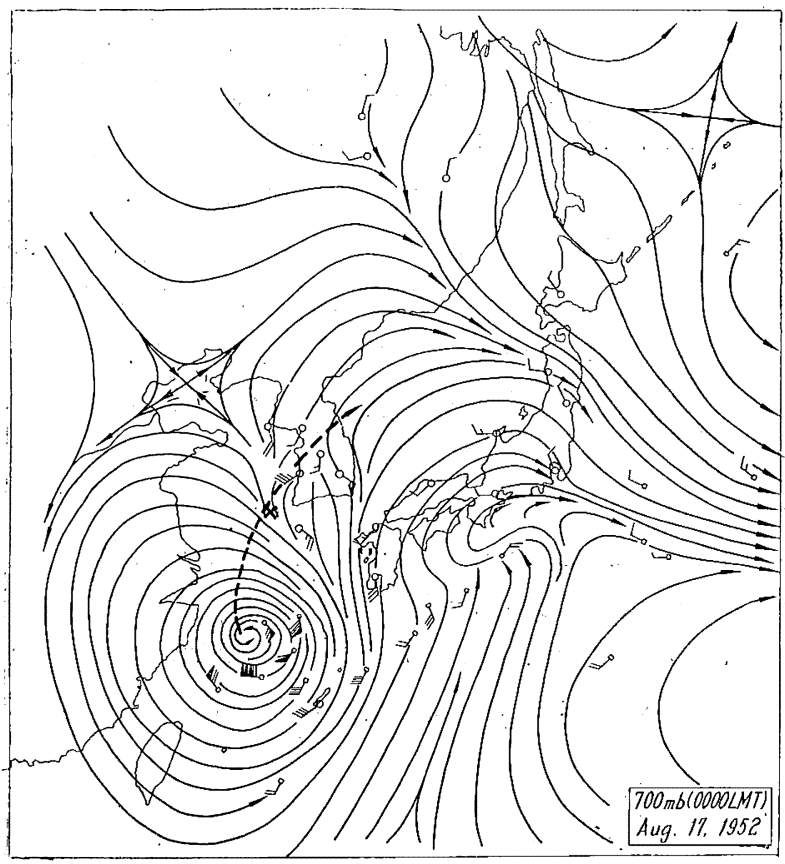

(b)

Fig. 3. Streamlines in the case of the typhoon Karen. The explanations for representation are same as in Fig. 1. inner side of this point streamlines direct towards the center with a cyclonic curvature and on the outer side they go out with an anticyclonic one. Therefore, the direction from the typhoon center to the delta point coincides with that of the steering current.

Further, as Fig. 1 shows, this delta point approaches towards the typhoon center with height. This fact, as shown in our previous report [8], is caused by the decrease of the cyclonic circulation of the typhoon with height. Therefore, at the level on which the typhoon circulation disappears, the delta point coincides with the typhoon center and the wind at this point becomes the steering current. This result perfectly agrees with that of NoRTON. Therefore, connecting the typhoon center and the delta points on each level, the steering current in the neighbourhood of the typhoon is obtained easily, and by this method the forecasting of typhoon movement is made possible:

In order to show the generality of this method, streamlines in the case of the typhoon Karen are shown in Fig. 3. In this example also, we may think that the 


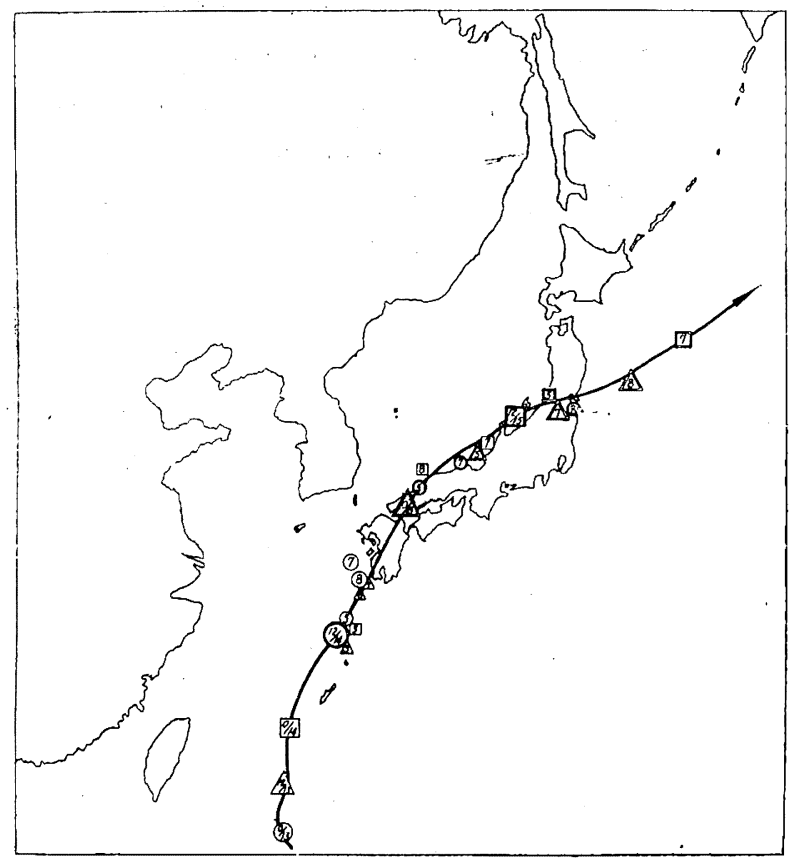

Fig. 4. The relation between the delta points and the typhoon track in the case of Ruth. typhoon moved towards the delta point.

In order' to indicate the: possibility of forecasting ty. phoon movent by means of the delta point which appears. in the streamline chart in the: case of a typhoon, the delta. points of each time and level, and the typhoon track in the case of the typhoon Ruth are shown in Fig. 4. In this figure, the heavy solid line represents the track of Ruth, denominators of fractional numbers. on the track represent date, mumerators time (in L. M. T.), and further, circles, triangles, squares ...... etc. surrounding: the fracitonal numbers and the

numbers 8,7 and 5 represent positions of the typhoon and the delta points at $850 \mathrm{mb}$, $700 \mathrm{mb}$ and $500 \mathrm{mb}$ level at the same time. As this figure shows, almost all the delta. points ride on the track, and for some cases, even the positions after 72 hours can. be forecasted. Of course, observational wind data are still insufficient, and sub. jective elements considerably remain in the drawing of streamlines. Therefore, in future, by repletion of observational wind data and the objective method for draw. ing of streamlines, this method for the forecasting of typhoon movement will be: developed.

Acknowledgement - The authors wish to express their hearty thanks to Dr. H. ARAKawa and the members of the Forecast Research Laboratory; Meteorological Research Institute, for their encouragement and helpful advice throughout the work. Sincere thanks are also due to Miss Y. Okubo for her indispensable aid in locating: the desired data and figures.

\section{References}

[1] Magata, M. 1950: Dynamics of the Movement of Atmospheric Vortices, Papers in Meteorology and Geophysics, 1, p. 38. 
[2] Syono, S. 1951: On the Motion of a Vortex in a Non-Uniform Pressure Field, Papers in Meteorology and Geophysics, 2, p. 1.

[3] Yoshitake, M. 1951: On the Movement of Typhoon (unpublished).

[4] Ootani, T. 1935: On the Forecasting of the Moving Direction of Typhoon by Upper Winds, Journal of the Meteorological Society of Japan, 13, p. 288 (in Japanese).

[5] Norton, G. 1944: Some Notes on Forecasting for Atlanta and Miami Districts, U. S. Weather Bureau Research Paper, No. 15.

[6] Sherman, L. 1950: On the Propagation of Hurricanes, Transaction of the American Geophysical Union, 31, p. 531.

[7] Hashimoto, M. 1951: On the Movement of Typhoon, Journal of Meteorological Research, 3, p. 242 (in Japanese).

[8] Masuda, Y. and TAKEUChI, M. 1951: An Aerological Investigation of the Structure of the Typhoon (Analysis of the Typhoon Jane and Kezia), Papers in Meteorology and Geophysics, 2, p. 231. 